\title{
Coopération agricole de production : quand l'activité agricole se distribue entre exploitation et action collective de proximité
}

\author{
Véronique Lucas ${ }^{1}$, Pierre Gasselin², Franck Thomas ${ }^{3}$, \\ Pierre-François Vaquié ${ }^{4}$
}

\section{Introduction}

De nouvelles formes d'action collective de proximité émergent en France entre les agriculteurs. À partir de modalités plus ou moins anciennes (coopérative, association, groupement d'employeurs, etc.), se constituent des groupes d'agriculteurs qui élaborent leurs projets de manière collective, de façon inédite avec peu de références, et parfois sans accompagnement institutionnel. Le réseau des coopératives d'utilisation de matériel agricole (Cuma) est un témoin privilégié de ces initiatives, ce qui a conduit leur Fédération nationale (FN Cuma) à engager depuis 2012, un travail de recherche-action pour mieux comprendre ce phénomène. Les premiers résultats constituent le cour de ce chapitre ${ }^{5}$.

Partant d'une expérience de mutualisation de matériel et/ou de travail, voire de salariat partagé, des groupes d'agriculteurs étendent les horizons de leur projet collectif et développent diverses initiatives : diversification des productions et/ou des activités, concertation des assolements, transformation et/ou commercialisation partagées, etc. Ces initiatives s'ouvrent parfois à de nouveaux acteurs tels que les collectivités territoriales. Cette reconfiguration des modalités et des objectifs de l'action collective impacte l'évolution des exploitations et interroge à bien des égards.

- Quelles sont ces nouvelles formes ? Peut-on leur reconnaître des caractéristiques communes?

1.FN Cuma/INRA, UMR 951 Innovation, 34875 Lattes, France, veronique.lucas@cuma.fr

2. INRA, UMR 951 Innovation, 34060 Montpellier, France, gasselin@supagro.inra.fr

3. FN Cuma, 75538 Paris, France, franck.thomas@cuma.fr

4. FN Cuma, 75538 Paris, France, pierre-francois.vaquie@cuma.fr

5. Ce travail, animé par la FN Cuma, a bénéficié du soutien du ministère de l'Agriculture (Casdar).

Ce chapitre de l'ouvrage L'agriculture en famille : travailler, réinventer, transmettre est publié en Open Access sous licence creative commons CC-BY-NC-ND permettant l'utilisation non commerciale, la distribution, la reproduction 
- Quelles motivations président à l'engagement d'agriculteurs dans de nouvelles formes d'exploitation et d'action collective? À quelles innovations techniques et organisationnelles correspond ce processus?

- Comment ces mutations impactent les frontières et le fonctionnement de l'exploitation? Correspondent-elles à des modèles agricoles particuliers ? Comment accompagner ces agriculteurs dans ces recompositions de l'action collective, autour de processus dynamiques et en l'absence de références stabilisées ?

Après un cadrage théorique et méthodologique, l'article aborde ces différentes questions en cherchant d'abord à décrire et caractériser ces nouvelles formes d'action collective de proximité entre les agriculteurs, et dans un second temps à interpréter leur motivation à s'y engager. Nous discutons ensuite des implications de ce mouvement dans l'évolution des logiques productives en agriculture ainsi que dans le développement agricole.

\section{Cadre d'analyse et méthodologie}

\section{L'action collective : un fait social inhérent à l'activité agricole}

Depuis le Néolithique, l'agriculture se transforme à travers des processus d'action collective. Pour stocker, produire, utiliser l'espace, gérer les ressources communes, échanger, faire face aux risques, les agriculteurs se sont organisés collectivement de diverses façons, de leur propre initiative ou sous l'impulsion d'autorités, en adoptant des règles communes construites de manière informelle ou instituées par le droit (Ostrom, 1990 ; Mazoyer et Roudart, 2002). En France, les lois d'orientation agricole de 1960 et 1962 qui " instituent " l'agriculture familiale, renforcent en parallèle l'agriculture de groupe, qu'elle soit structurée de façon coopérative (coopératives d'approvisionnement et de collecte, Cuma), associative (groupes de développement) ou sociétaire (groupement agricole d'exploitation en commun, groupement foncier agricole). Ces organisations constituent des outils de facilitation de la mise en place dans les années 1960 et 1970, d'un modèle agricole familial productiviste (Nicolas, 1988 ; Lefevre, 1996 ; Barthez, 2000 ; Lucas, 2005).

Les vingt-cinq dernières années ont été marquées par un double mouvement combiné de renforcement de la tendance modernisatrice et d'hétérogénéité croissante de l'agriculture, devenue plurielle sous l'effet d'une diversification des logiques productives et commerciales, ainsi que des fonctions exercées (Allaire et Boyer, 1995 ; Hervieu, 2002). Cette fabrique de nouvelles agricultures (Lémery, 2003), marquées par une plus grande hétérogénéité dans le contexte de sociétés en réseaux, explique les recompositions actuelles de l'action collective en France.

D'une part, on peut citer l'émergence de nouveaux collectifs dans le champ des agricultures alternatives, caractérisés par la figure du réseau (comme le Réseau Semences Paysannes, ou Biodiversité, Agriculture, Sols \& Environnement [BASE]). 
Ces nouvelles configurations sociotechniques, entre agriculteurs distants géographiquement, visent la production et le partage de connaissances (Demeulenaere et Goulet, 2012).

D'autre part, on peut citer le mouvement actuel de concentration des coopératives de collecte et d'approvisionnement, dont les relations avec les adhérents sont marquées par un phénomène de distanciation (Touzard et Draperi, 2003 ; Filippi et al., 2009 ; Barraud-Didier et al., 2012).

\section{Proximité géographique et de coordination}

Les recompositions actuelles de l'action collective se manifestent également au sein des formes d'organisation de proximité entre agriculteurs. Nous nous intéresserons à celles visibles à partir du réseau des Cuma, correspondant à des collectifs mêlant différentes modalités d'arrangement et d'organisation, en plus de la structure juridique de la Cuma. Au vu de leurs caractéristiques, nous proposons de les qualifier d'action collective de proximité (Bouba-Olga et Grossetti, 2008).

Premièrement, la proximité géographique entre les acteurs de ces formes sociales est induite par la matérialité des ressources mutualisées (intrants, équipements, infrastructures, travail, salariés, productions, foncier) qui impose un certain degré de proximité physique, voire de contiguïté spatiale entre les exploitations.

Deuxièmement, la mutualisation de ces ressources est rendue possible par un niveau minimal d'interconnaissance et de confiance mutuelle qui rend possible l'engagement de chaque agriculteur dans la dynamique collective de partage. Ces formes sociales sont donc encastrées dans les réseaux socioprofessionnels locaux, qui génèrent une proximité socio-économique (de ressource et de coordination) entre les agriculteurs impliqués. En effet, dans le champ professionnel agricole, ce type de réseaux présente des caractéristiques particulières comme différents auteurs l'ont démontré.

Ainsi, Darré (1996) souligne que les agriculteurs cherchent, à travers des interactions avec leurs pairs, à développer des dialogues techniques qui leur permettent d'enrichir leur travail réflexif visant à adapter leurs pratiques. La morphologie des réseaux socioprofessionnels locaux détermine la densité et la qualité des dialogues entre les agriculteurs d'un même territoire, à travers lesquels se jouent des dynamiques d'inter-influence contribuant à évaluer les pratiques en vigueur et ainsi à les " normer ". Ces réseaux qui tendent à produire des représentations communes de la réalité formant un système de normes local, sont donc à la fois habilitants et contraignants pour les agriculteurs (Giddens, 1987).

Sabourin $(2007,2012)$ met en évidence que l'action collective entre agriculteurs ne met pas en jeu seulement des besoins matériels, mais aussi des liens sociaux et symboliques. Selon lui, on ne peut réduire par exemple les prestations de travail dans les chantiers en commun à de simples échanges de services. D'ailleurs, le droit français reconnaît dans l'entraide une relation de réciprocité dans l'acte de production agricole et non pas une relation d'échange marchand (Code rural, articles L.325-1 
et suivants). Par conséquent, ce type d'interactions génère des valeurs humaines et éthiques, positives ou négatives, exprimées par les agriculteurs à travers l'évocation de sentiments d'obligation ou de solidarité, d'appartenance ou de distanciation, ou encore de confiance. Ces valeurs constituent des produits, ainsi qu'un moteur ou un frein (selon qu'elles soient positives ou négatives) au renouvellement des cycles de réciprocité.

Ces systèmes de normes et de valeurs générés par les réseaux socioprofessionnels locaux en agriculture constituent des ressources cognitives qui facilitent ou handicapent la coordination de proximité entre agriculteurs.

Troisièmement, ces facteurs de proximité sont renforcés par des ressources de médiation, constituées par les conventions partagées qui se cristallisent dans les règles communes adoptées par les collectifs. Une partie d'entre elles est construite par les agriculteurs impliqués, tandis que d'autres sont données de manière institutionnelle à travers les statuts juridiques des structures mobilisées dans l'action collective. Des agencements à chaque fois particuliers s'élaborent entre les diverses modalités formelles et informelles de coordination et de mutualisation engagées.

Quatrièmement, une partie de ces formes sociales implique d'autres acteurs externes à l'agriculture, notamment des collectivités locales. Ce caractère multi-acteurs contribue à renforcer la dimension territoriale, qui interagit avec les autres effets de proximité caractéristiques de ces dynamiques socioéconomiques.

Pour résumer, ces formes d'action collective de proximité sont agencées par les agriculteurs en mobilisant des processus sociotechniques, qu'ils régulent de façon flexible en s'appuyant sur des systèmes de normes, de règles et de valeurs ainsi que sur des institutions juridiques, et ceci de manière plus ou moins ancrée au territoire.

\section{Un processus de recherche-action}

Le travail d'étude dont nous présentons ici les résultats est né d'une réflexion de la FN Cuma, qu'elle a choisi de partager et d'approfondir avec un ensemble de partenaires du développement agricole et de la recherche ${ }^{6}$. Constatant un questionnement et des recompositions de l'action collective de proximité par une partie des adhérents, ses responsables ont décidé d'orienter l'action du réseau fédératif pour appuyer l'émergence et le développement des diverses formes de coopération entreprises par les agriculteurs autour de leur activité productive. Pour caractériser ces dernières, la FN Cuma a avancé le concept opératoire de coopération agricole de production, défini comme « l'ensemble des pratiques de coopération ayant pour objet la production agricole » (FN Cuma, 2012a, 2012b ; Lacam et al., 2012).

6. Coop de France, CERFRANCE, Fédération nationale d'agriculture biologique (FNAB), Fédération nationale des centres d'initiatives pour valoriser l'agriculture et le milieu rural (FN Civam), Gaecs et Sociétés, Trame, Confédération générale des sociétés coopératives et participatives, Institut national de la recherche agronomique, ministère de l'Agriculture. 
Afin de mieux percevoir les implications de son nouveau positionnement, la FN Cuma a initié un processus réflexif avec des organisations partenaires concernées par le renouvellement de l'action collective de proximité entre agriculteurs, en y associant la recherche. Un cycle de journées d'étude s'est concrétisé en 2012 et 2013 afin de partager une analyse commune de ce phénomène. Il a permis d'identifier différents traits et enjeux des reconfigurations actuelles de l'action collective de proximité en agriculture, permettant d'avancer des hypothèses explicatives. Cette étude d'un tissu social particulier, celui des pratiques de coopération articulées autour des Cuma, révèle des processus sociotechniques inédits au sein de l'agriculture française.

\section{Méthodologie}

Le contenu du cycle réflexif fournit les matériaux principaux de notre analyse, articulés autour (i) de monographies d'une quinzaine de cas étudiés (FN Cuma, 2012a ; Lacam et al., 2012 ; Lucas, 2013), constituées à partir d'entretiens collectifs semi-directifs et (ii) de différents travaux académiques en sciences sociales mis en discussion (Gasselin et al., 2012a, 2012b et voir aussi Gasselin et al. dans cet ouvrage ; Allaire et al., 2013 ; Cordellier, 2014). Par ailleurs, des acteurs de têtes de réseaux et des porteurs d'expériences de l'agriculture de groupe ont présenté des analyses issues de leurs organisations (Séronie et Boullet, 2007 ; de Torcy et Pommereul, 2012 ; Lurois, 2012 ; Moraël, 2012). Les journées ont réuni une large palette d'acteurs et de chercheurs à partir d'un agenda thématique séquencé faisant progresser un questionnement collectif. Au cœur de ce processus réflexif, la notion de coopération agricole de production a fourni un concept de travail qui a constitué un objet intermédiaire du dialogue entre chercheurs et acteurs. Ce dernier a permis aux participants de contribuer à nommer la nouveauté et a nourri un exercice sémantique pour interroger les fondamentaux des formes observées, synthétisé par des écrits intermédiaires entre chaque journée. En complément, la tenue d'un colloque national à mi-parcours (fin 2012) a permis de divulguer l'état des réflexions et de les préciser au vu des réactions suscitées. Enfin, un travail collectif de construction d'un projet de recherche-développement pour poursuivre ce processus réflexif a demandé la réalisation de travaux exploratoires supplémentaires, notamment une revue bibliographique thématique (FN Cuma, 2013). La frise ci-après récapitule l'ensemble de cette démarche de travail' .

7. Les documents relatifs au cycle réflexif sur la coopération agricole de production sont inclus dans la bibliographie et disponibles par ce lien : http://www.cuma.fr/france/content/cooperationagricole-de-production [consulté le 21/07/2014]. 


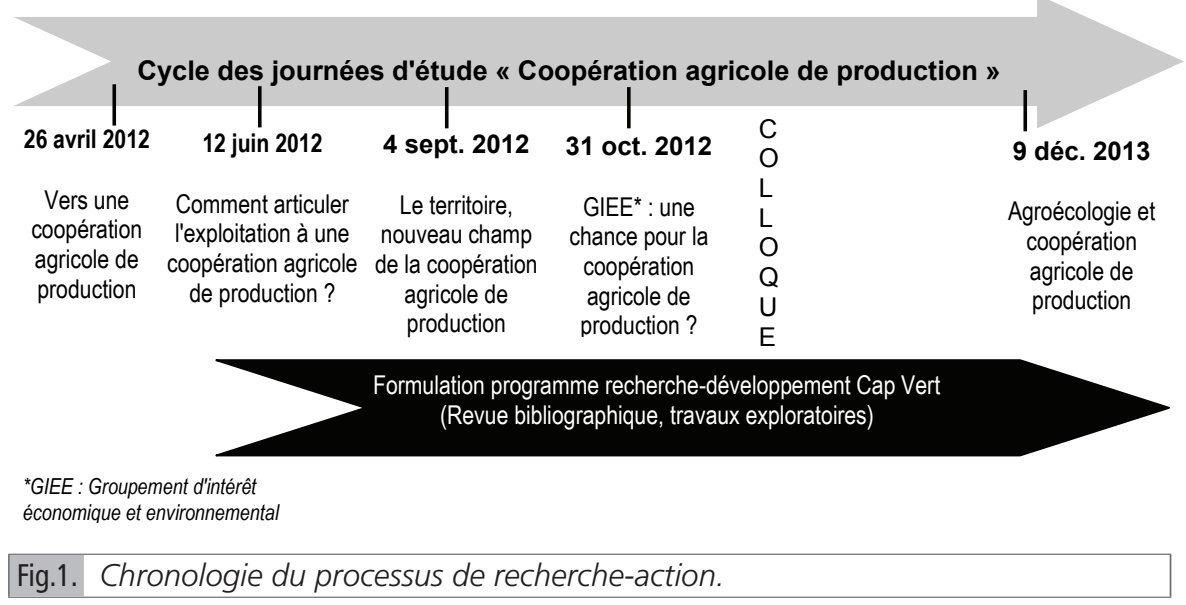

\section{Caractéristiques des formes de coopération agricole de production}

Pour donner un aperçu de ce que peut recouvrer la coopération agricole de production, le tableau 1 détaille six cas étudiés, choisis pour leur diversité, parmi la quinzaine mobilisée durant le cycle réflexif.

\section{Un empilement des formes organisationnelles et des statuts juridiques}

La coopération agricole de production correspond à des formes sociales qui se traduisent par des choix techniques communs entre les agriculteurs impliqués, une propriété en partie collective voire impartageable d'équipements, d'infrastructures, voire de foncier, l'interconnaissance et la proximité spatiale et socioéconomique de leurs membres, des dynamiques de partage matériel et immatériel (compétences), monétaires et non monétaires, ainsi que des processus de concertation réguliers, et parfois continus. Pour certains des cas étudiés, la mutualisation poussée conduit à l'organisation de nouvelles formes d'exploitation où plusieurs agriculteurs (parfois une dizaine) conduisent ensemble une partie de leurs activités. Des pans entiers de l'activité de production des exploitations participantes s'interpénètrent ainsi sans forcément fusionner. Ceci repose sur une délicate articulation entre mise en place d'un outil commun, construction d'un ou plusieurs collectifs, préservation des stratégies individuelles, voire inscription dans un projet de territoire.

Ces formes sociales s'accompagnent d'innovations et hybridations organisationnelles, aboutissant à de nouvelles configurations de proximité. Les agriculteurs impliqués combinent des outils juridiques variés (Cuma, groupement d'employeurs, Groupement d'intérêt économique, etc.), voire des arrangements plus informels (banque de travail, entraide, groupement d'achat, etc.). 


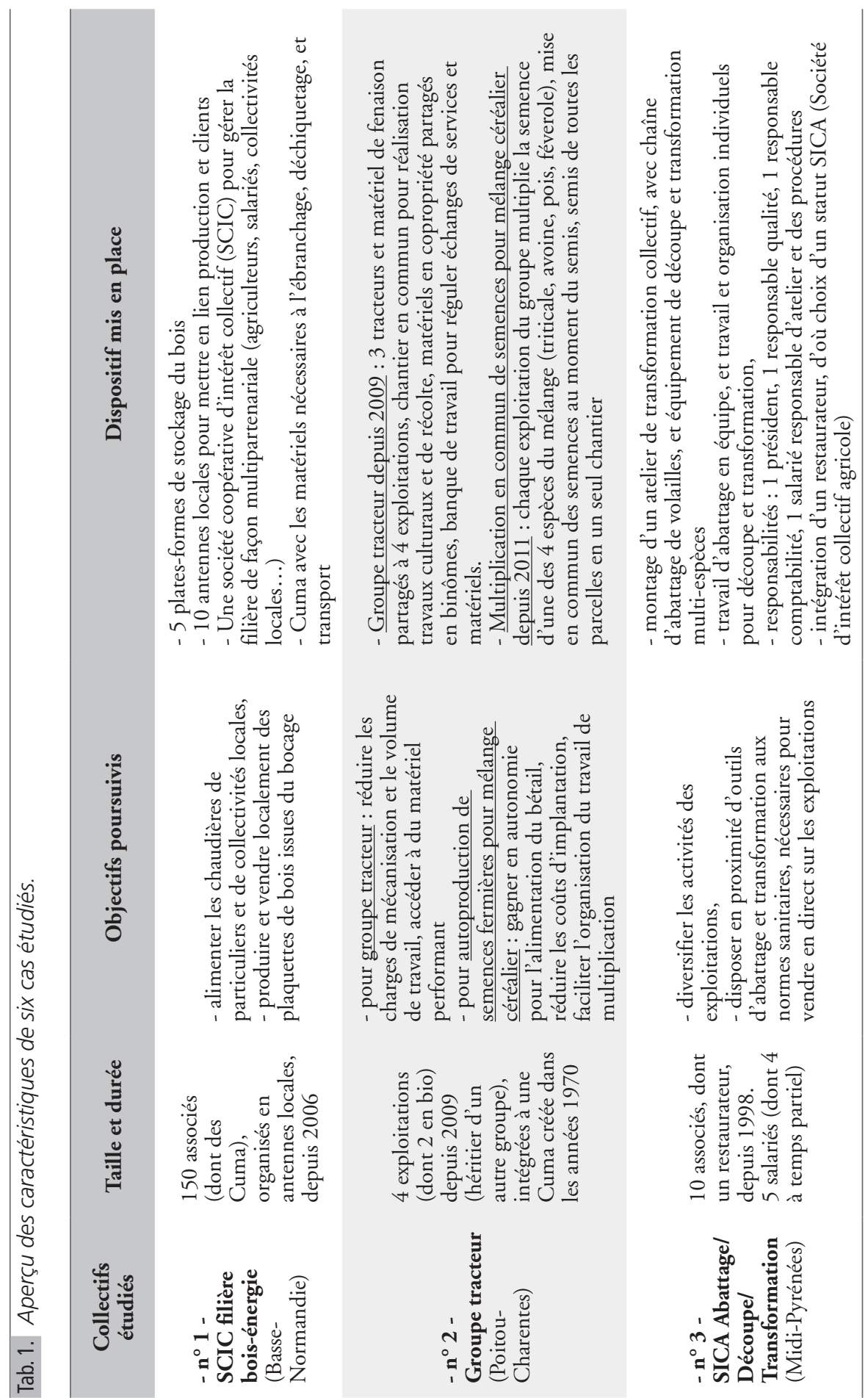




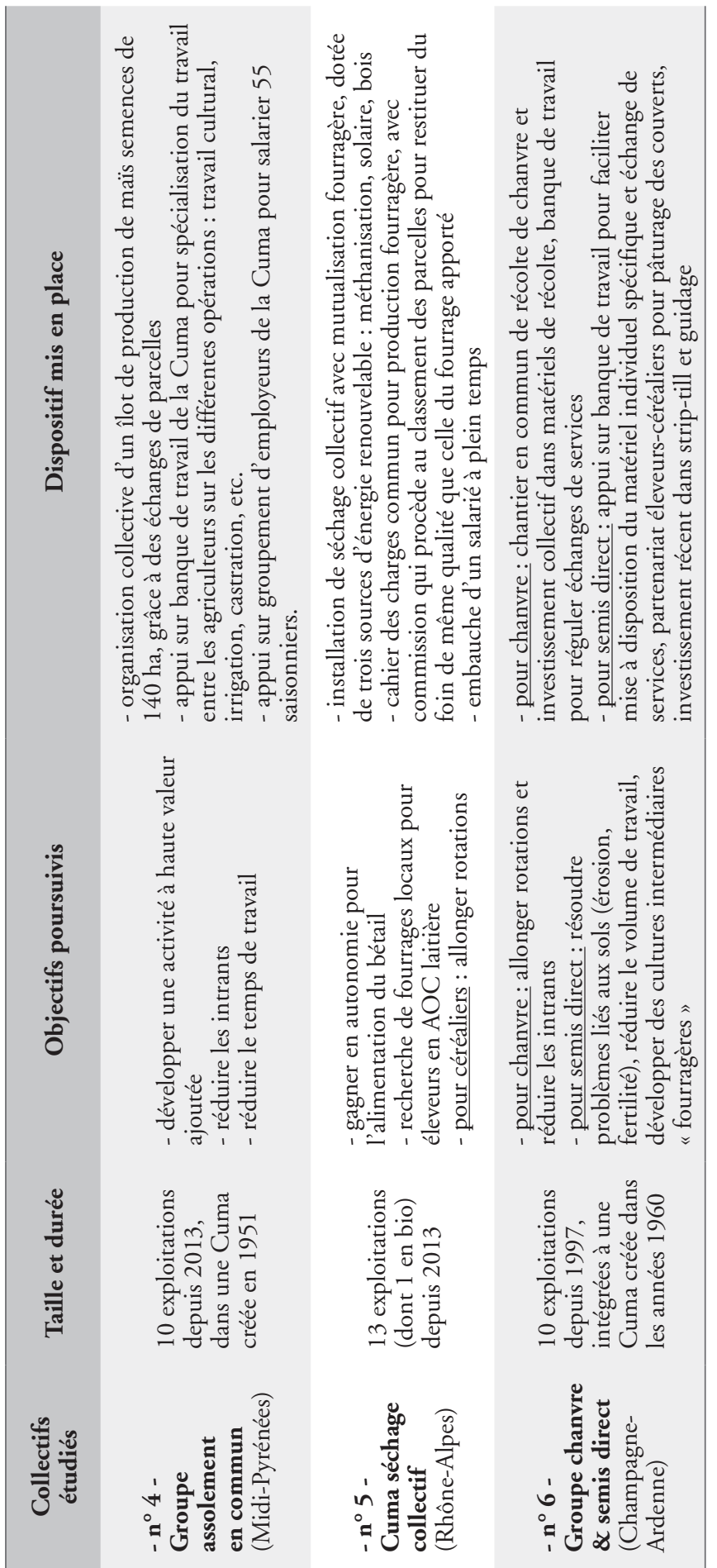




\section{Un jeu d'acteurs qui ancre l'action collective au territoire}

Ces formes sociales se constituent principalement via la mutualisation, par les agriculteurs impliqués, de tout ou partie de leurs stratégies de production, au travers du matériel, du travail, de salariés, du foncier, des intrants, des bâtiments, etc. Dans un contexte d'instabilité avec une faible vision de l'avenir, une partie des agriculteurs impliqués cherche à ne pas figer leur organisation pour être flexibles et réactifs, et ainsi garder la possibilité d'évoluer selon les aléas de la conjoncture (Gasselin et Bathfield, 2013).

La coopération agricole de production repose sur diverses logiques de proximité, où différentes alliances émergent entre les exploitations, et plus particulièrement entre leurs activités productives. Ainsi, ces formes sociales ne relèvent pas de schémas standardisés, mais sont le produit d'une construction progressive de liens entre les acteurs d'un même territoire, agricoles et parfois non-agricoles. Les cas étudiés montrent qu'elles résultent d'une ou plusieurs histoires longues de coopération de proximité, avec souvent des étapes premières où les agriculteurs participants ont d'abord expérimenté des coordinations exigeant un faible engagement au sein du groupe. Les expériences permettent aux agriculteurs d'affirmer progressivement leurs implications. Ces trajectoires enracinent ces formes sociales dans le territoire où elles se déroulent.

\section{Une géométrie variable propre aux organisations en réseau}

L'une des caractéristiques fortes de ces formes sociales réside dans leurs géométries variables et l'absence de cadre normatif. La coopération agricole de production n'est pas une méta-organisation au périmètre résultant de la simple agrégation de trois, sept ou dix exploitations. Les fonctions partagées et les acteurs engagés ne sont pas donnés a priori, et une même exploitation participe en même temps à plusieurs organisations. Se dessine ainsi l'image d'un tissu d'exploitations avec différents types de connexion entre elles, révélant les traits caractéristiques d'un fonctionnement en réseau : il n'existe pas de décisions centralisées, mais une pluralité d'espaces de concertation qui atténue les frontières de l'exploitation. L'appartenance à ces formes d'organisation tient à l'engagement même des acteurs, et ce fonctionnement interroge la gouvernance des structures instituées.

La construction de ces formes sociales résulte moins d'une planification que d'une accumulation de micro-organisations : chaque élément est déterminé par la volonté des acteurs mais la conscience de l'ensemble n'est pas forcément partagée.

\section{Des complémentarités parmi l'hétérogénéité des systèmes productifs}

Ce mode de coopération agricole de production conduit ainsi à mettre en relation des systèmes productifs différents : des agriculteurs conventionnels côtoient voire 
investissent avec des agriculteurs en bio, des viticulteurs s'engagent sur un emploi partagé avec des éleveurs... Au détour d'un matériel en commun, d'un groupement d'employeurs ou d'un point de vente collectif, se découvrent des proximités et des complémentarités entre des projets d'exploitation aux stratégies distinctes.

À partir d'une exploitation impliquée dans le cas étudié $n^{\circ} 2$ (Tab. 1), nous avons schématisé les relations de coopération multiples entre exploitations de ce territoire (Fig. 2).

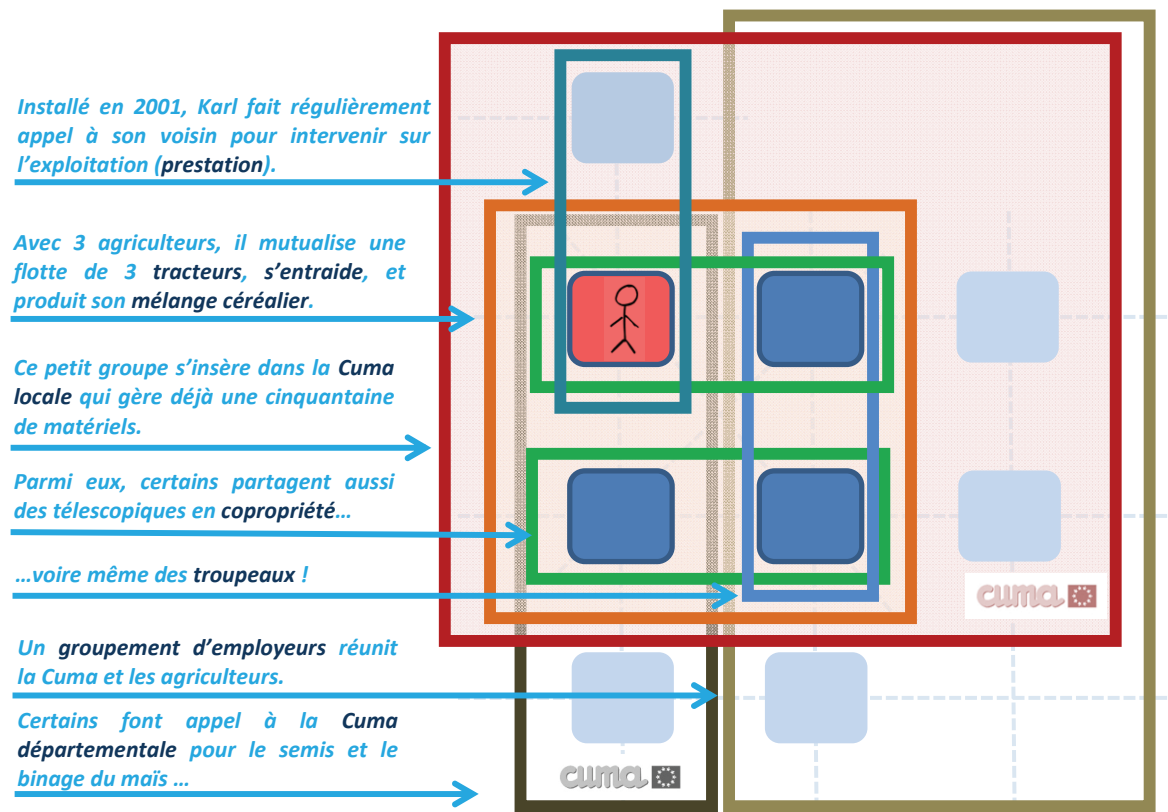

Fig. 2. Exemple d'une forme de coopération agricole de production sur un territoire (cas étudié $n^{\circ} 2$ ).

Même si nos travaux permettent de dégager des traits communs à ces organisations collectives, la première de leurs caractéristiques reste l'extrême diversité des formes constatées, notamment conditionnée par l'histoire et les organisations préexistantes sur le territoire, ainsi que les objets mis en commun par les acteurs.

\section{De nouvelles coopérations pour soutenir le changement}

L'objet de ces formes de coopération agricole de production est multiple, mais nous repérons qu'il vise le plus souvent à faciliter la construction de nouvelles pratiques et systèmes d'exploitation. 


\section{La " fabrique » de nouvelles agricultures}

Depuis plus d'une vingtaine d'années, les activités agricoles sont mises à l'épreuve par de nouvelles demandes adressées à l'agriculture, ainsi que par les limites écologiques et économiques qu'elles rencontrent. Cette crise radicale qui ne peut être abordée par les agriculteurs simplement en termes d'adaptation, induit une part croissante d'inventions, notamment via l'exploration de nouvelles façons de faire et la redéfinition de leurs rôles (Lémery, 2011). Nous repérons au moins cinq facteurs actuels de mutation des systèmes d'activités :

- la recherche de solutions agronomiques : il s'agit d'agriculteurs confrontés à des impasses agronomiques, et/ou une plus grande fréquence des accidents climatiques. Pour réagir à la moindre efficacité des intrants chimiques (résistances aux produits phytosanitaires par exemple), aux problèmes d'érosion et de baisse de la fertilité des sols, ils cherchent à diversifier les rotations en introduisant de nouvelles espèces, à recourir davantage au désherbage mécanique et à la fertilisation organique, à diminuer le travail mécanique du sol, etc. (Fleury et al., 2011 ; Ricci et al., 2011 ; Meynard et al., 2013). Pour faire face à la variabilité climatique, des éleveurs mettent en œuvre des stratégies de diversification de leurs productions fourragères (Nil, 2012 ; Figureau, 2013) ;

- une meilleure productivité du travail : l'agrandissement des exploitations, les stratégies de diversification, l'aspiration à dégager du temps libre incitent des agriculteurs à revoir l'organisation du travail. Cette orientation peut entraîner un moindre travail cultural, ou une spécialisation du travail par champs de compétences, ou la mécanisation de certaines tâches, ou une simplification poussée des systèmes productifs, etc. (Charroin et al., 2012 ; Hostiou et Fagon, 2012 ; Quentin, 2012) ;

- de nouvelles stratégies économiques : le contexte économique marqué par la concentration des acteurs de l'aval, et depuis 2007 par la volatilité des prix des intrants, incite les agriculteurs à de nouvelles stratégies pour maîtriser leurs charges, commercialiser dans de nouveaux circuits, développer d'autres activités génératrices de revenu, etc. Ainsi, des éleveurs manifestent leur volonté de devenir plus autonomes pour l'alimentation de leurs animaux, tandis qu'un producteur sur cinq vend aujourd'hui en circuit-court (Barry, 2012 ; Garambois et Devienne, 2012) ;

- le souci environnemental : un nombre croissant d'agriculteurs s'engage dans des pratiques plus respectueuses de l'environnement (agriculture biologique par exemple), par raisonnement économique, pour des raisons de santé, par éthique personnelle (notamment de la part des nouveaux entrants non issus du milieu agricole, qui représentent une part croissante des installations), etc. (Lamine et Bellon, 2009 ; Lefebvre, 2009);

- la multiplication des normes : une diversité de systèmes normatifs impose aux agriculteurs des changements pratiques, tels que les régulations environnementales publiques, l'émergence de labels et marques privés dans un objectif d'affichage environnemental, l'évolution des cahiers des charges de l'appellation 
d'origine contrôlée (AOC) vers un lien au terroir plus affirmé (plus grande autonomie alimentaire à l'échelle de la zone d'appellation pour les AOC d'élevage par exemple), etc. (Hirczak, 2007 ; Bonnieux, 2009 ; Lucas, 2012).

Ces différents facteurs conduisent à une hétérogénéité des logiques productives entre agriculteurs (Ploeg et al., 2009 ; Le Guen, 2011 ; Lémery, 2011).

\section{L'action collective, levier de l'innovation}

Nous avons identifié au moins trois raisons principales qui conduisent les agriculteurs à se tourner vers la coopération agricole de production pour engager ces nouvelles pratiques.

\section{Des logiques d'investissement différentes}

Des pratiques induisent des investissements spécifiques, par exemple dans de nouveaux équipements qui s'ajoutent en se substituant peu à ceux déjà détenus (cas des matériels pour le désherbage mécanique, le non-labour, la transformation fermière, etc.). Cette tendance, à rebours de la logique de spécialisation, est renforcée par les stratégies de diversification des productions et des activités sur une partie des exploitations qui tendent à renchérir les charges structurelles. La possibilité d'investir collectivement dans un parc matériel élargi d'équipements spécifiques sécurise l'engagement dans le changement de pratiques et la création de nouvelles activités, quand elle n'en devient pas une condition indispensable.

\section{L'inadéquation du régime sociotechnique actuel}

Les agriculteurs sont aujourd'hui confrontés à un manque de solutions et de conditions adaptées de la part de l'ensemble constitué par l'agrofourniture, l'agroalimentaire et la distribution, ainsi que l'appareil de recherche-développement (Rip et Kemp, 1998 ; Vanloqueren et Baret, 2009). Ceci les conduit à coopérer pour résoudre leurs questions par eux-mêmes. D'une part, l'action collective permet à des agriculteurs d'accéder à des ressources stratégiques qu'ils trouvent difficilement ou de manière peu satisfaisante auprès des opérateurs de l'agrofourniture. C'est le cas des producteurs s'organisant pour la production et l'échange de semences fermières, ou de ceux qui se coordonnent avec un équipementier pour co-concevoir des matériels adaptés à leurs conditions spécifiques de travail (pour les outils de semis direct par exemple) (Lucas, 2012). D’autre part, des coopérations deviennent nécessaires pour assurer la viabilité économique des nouvelles pratiques sans débouchés satisfaisants dans les filières existantes. Ainsi, différents cas de relance de cultures de diversification (chanvre, lin, luzerne, etc.) ont été facilités par l'organisation de collectifs de producteurs en Cuma ou groupes de développement, pour maîtriser et gérer ces nouvelles activités (Meynard et al., 2013). Dans d'autres cas, la Cuma permet le maintien de productions menacées par exemple par la concentration des entreprises agroalimentaires, avec qui le rapport de forces devient plus défavorable 
pour les producteurs. Des groupes investissent en commun dans des équipements de stockage et de conditionnement, voire de transformation pour commercialiser en circuits courts (Mondy et al., à paraître ; Mundler et al., à paraître). Par ailleurs, la coopération de proximité favorise l'émergence de coordinations pour le recyclage de coproduits, par exemple pour l'alimentation du bétail (production de tourteaux grâce à l'investissement dans une presse à huile), ou pour la fertilisation organique (organisation collective de co-compostage, méthanisation territoriale pour obtenir un digestat fertilisant, voire mieux valoriser les engrais verts) (Pierre, 2013). Ce type de stratégies collectives pour concevoir des solutions adaptées aux situations des agriculteurs contribue à l'innovation dans le contexte d'un régime sociotechnique devenu inadéquat.

\section{L'impasse individuelle}

Certaines nouvelles pratiques nécessitent d'agir à une échelle dépassant celle de l'exploitation. Par exemple, des coopérations à l'échelle d'un terroir ou d'un bassin versant permettent des actions concertées entre agriculteurs au niveau des processus écologiques (Léger, 2013). Ainsi, des producteurs se coordonnent pour procéder chaque année à des échanges de parcelles pour éviter le recours trop fréquent de certaines cultures au même emplacement, ce qui devient problématique dans un contexte de moindre efficacité des intrants chimiques. De même, des producteurs de semences organisent des démarches collectives de pollinisation dirigée.

Ces formes d'organisations développent de nouvelles fonctions qui contrastent avec le rôle longtemps joué par la Cuma pour faciliter la mise en œuvre des techniques proposées par le régime sociotechnique dominant (Lefevre, 1996). Aujourd'hui, s'opèrent de manière croissante dans les Cuma et groupes de développement des processus de co-conception de solutions, tant d'ordre techniques qu'organisationnelles, en raison de la limitation de connaissances valides et actionnables pour soutenir ces nouvelles pratiques et activités. Par la coopération agricole de production, l'agriculteur invente et conçoit des pratiques et modes d'organisation, souvent fondés sur les atouts et contraintes de son environnement agroécologique et territorial. Cet exercice de conception est facilité par le dialogue entre pairs, dont le regroupement favorise également le partenariat avec des acteurs externes.

\section{À la croisée des modèles de développement agricole}

\section{Des recompositions dans l'ensemble de l'agriculture}

La reconfiguration actuelle de l'action collective de proximité pour faire évoluer les pratiques et systèmes d'exploitation s'observe dans l'ensemble du paysage agricole français, dont on constate une polarisation entre deux tendances principales (Garambois et Devienne, 2012 ; Ploeg, 2014). Il s'agit d'une part, d'une tendance marquée par l'expansion continue de structures d'exploitation de grande taille, 
associée à des gains de productivité physique du travail, rendue possible par le recours important aux intrants et capitaux externes qui se substituent au travail. L'autre tendance correspond à la recherche de valorisation des potentialités propres des exploitations, de manière endogène, à partir de stratégies de diversification, d'autonomisation, et/ou de création de valeur ajoutée. Entre ces deux pôles, et comme le fait remarquer Ploeg (2014), coexistent de " larges zones grises " induites par les fluctuations et hybridations qui caractérisent les trajectoires et stratégies des agriculteurs, ainsi que les actions collectives auxquels ils s'intègrent.

\section{Du côté de la tendance " expansionniste " des structures de grande taille}

De nouvelles modalités de coopération émergent entre agriculteurs pour franchir de nouveaux caps en matière de gains de productivité du travail, en repoussant la limite du nombre d'hectares ou d'animaux qu'un actif agricole peut prendre en charge. Assolements concertés, maternités collectives en production porcine, regroupement de troupeaux, délégation de certaines tâches à travers du salariat partagé, associé ou non à des activités organisées en commun (par exemple la reprise et distribution des fourrages en élevage) permettent d'aller plus loin dans la concentration productive, la simplification des systèmes productifs, la mécanisation et l'automatisation du travail déjà entreprises à l'échelle des exploitations (Séronie et Boullet, 2007 ; Cochet, 2008 ; Charroin et al., 2012).

\section{Du côté de la tendance " endogène "}

Ploeg (2014) note que les agriculteurs orientés vers la diversification et la recherche d'économies, activent de nouveaux modes de coordination entre eux, pour substituer certaines transactions marchandes par des formes d'organisation ou de prestations réciproques. Cette préférence s'expliquerait notamment, selon l'auteur, par la méfiance engendrée par des comportements opportunistes d'acteurs marchands, lesquels conduisent les agriculteurs à privilégier la coopération entre pairs pour accéder à des ressources stratégiques pour leurs systèmes productifs (échange de semences fermières par exemple).

Ces actions collectives, confortant des stratégies productives plus fortement génératrices d'emploi et de valeur ajoutée sur leurs territoires (Garambois et Devienne, 2012 ; Mahé et Lerbourg, 2012 ; Ploeg, 2014), rencontrent une considération plus favorable de certaines collectivités territoriales. Une partie des élus politiques devient plus consciente des potentialités économiques de formes d'agriculture valorisant les ressources locales, tout en cherchant à reprendre la main sur des domaines jusque-là gouvernés par des politiques centralisées et/ou des opérateurs économiques a-territoriaux, comme le montrent les exemples du secteur de l'énergie et de la restauration scolaire (Fiamor, 2011 ; Le Velly et Bréchet, 2011 ; Tritz, 2012 ; Pierre, 2013). 


\section{Au sein des " zones grises "....}

Certains cas étudiés attestent de l'élaboration de dispositifs techniques et organisationnels qui permettent à la fois des gains de productivité par des investissements collectifs et des gains d'autonomie par le développement de pratiques diminuant le recours aux intrants externes. Lexemple du cas $n^{\circ} 5$ (Tab. 1) illustre cette hybridation entre logique de substitution du travail par le capital et logique endogène : l'investissement de près de deux millions d'euros dans une installation collective de séchage en grange, partagée entre 13 exploitations, permet de développer la culture de prairies riches en légumineuses, et donc économes en intrants.

Par ailleurs, l'histoire récente du développement agricole montre la reprise de certaines innovations collectives par le régime sociotechnique dominant. Le développement actuel de la méthanisation territoriale, du co-compostage de déchets verts et effluents d'élevage, ou la relance de certaines filières comme celle du chanvre ou du bois-énergie ont été initiés grâce aux efforts passés de regroupements d'agriculteurs pionniers, en Cuma ou groupes de développement. Ayant passé le stade de l'expérimentation, ces nouveaux champs d'activité constituent aujourd'hui des opportunités rentables pour des opérateurs ou investisseurs externes à l'agriculture. Ceux-ci les saisissent pour installer des dispositifs industriels, dans lesquels les agriculteurs ne sont plus que de simples fournisseurs, dont l'activité devient partiellement subordonnée aux prérequis de ces nouvelles filières (Le Guen et de Casinière, 2010 ; Meynard et al., 2013).

\section{Quel cadre donner à la coopération agricole de production ?}

Parmi les nombreuses reconfigurations de l'action collective de proximité en agriculture, que peut-on considérer comme relevant de la coopération agricole de production?

\section{Les implications du principe coopératif}

Le concept opératoire de coopération agricole de production va au-delà de la notion de collaboration à une action commune, en introduisant des principes de coopération. Les mouvements de l'économie sociale déclinent ces principes à travers différentes règles, comme l'engagement volontaire et la participation effective aux décisions, le fonctionnement démocratique (une personne = une voix), la solidarité entre coopératives et avec le territoire, etc. (ACI, 1996 ; Draperi, 2011). L'ensemble de ces notions est repris et décliné dans le projet politique de la FN Cuma comme constitutif d'un modèle de développement à visée émancipatrice, préservant l'autonomie de décision et d'action de chaque agriculteur, et contribuant au développement des territoires, notamment en cherchant à installer et maintenir un grand nombre d'agriculteurs (FN Cuma, 2012b). 


\section{Asymétries et sélection}

Ces principes excluent donc de la coopération agricole de production, les formes sociales basées sur des modalités d'organisation asymétriques entre agriculteurs conférant une position de "donneur d'ordre » ou de " prescripteur » à l'un des protagonistes de la collaboration. En effet, de nouveaux types d'arrangement émergent entre agriculteurs, en renouvelant par exemple des formes de contrats d'intégration qui peuvent tendre à déposséder certains producteurs de la maîtrise de leur activité. Il peut s'agir par exemple de délégation de cultures annuelles sur les terres d'autres exploitations, à travers un cahier des charges indiquant les modalités culturales de l'implantation à la récolte, voire à travers une conduite de l'intégralité du cycle cultural (voir Anzalone et Purseigle dans cet ouvrage). Cochet (2008) observe que ces arrangements s'organisent surtout avec des agriculteurs désavantagés, qui tendent à perdre la maitrise de l'acte de production.

Plus largement, considérer la dimension coopérative de ces formes sociales amène à s'interroger sur les impacts de cette recomposition de l'action collective de proximité sur les processus de différenciation aujourd'hui visibles dans le champ professionnel agricole : la coopération agricole de production renforce-t-elle ou limite-t-elle la sélection générée par les mutations sociotechniques actuelles ? (Le Guen, 2011). Autrement dit, les formes sociales étudiées correspondent-elles au regroupement des seuls agriculteurs dotés d'un minimum de ressources culturelles, sociales et relationnelles pour conduire des processus d'innovation de manière autonome et maîtrisée ? Ou bien ouvrent-elles l'accès à la possibilité d'innover à un maximum d'agriculteurs?

\section{Repenser l'action publique}

Ces mutations de l'action collective de proximité en agriculture questionnent les cadres actuels des politiques publiques, notamment leur focalisation sur l'exploitation agricole. Elles invitent notamment à examiner l'effacement de la famille dans les solidarités de proximité, à interroger les statuts juridiques de la coopération agricole, et à repenser les modalités d'accompagnement.

\section{L'action collective de proximité réinventée : un substitut à la famille ?}

Ces formes sociales signent une dissociation des liens entre la terre, le travail et le capital, autrefois fondements de l'exploitation agricole familiale (Cochet, 2008). Peut-on dire que la coopération agricole de production reprend aujourd'hui certaines des fonctions exercées pendant longtemps par la famille agricole ? Cette dernière fut autrefois vectrice d'une cohésion sociale de proximité et structurante des processus d'entraide. Après les lois d'orientation de 1960-1962, elle a continué à jouer un rôle important dans l'agriculture, notamment dans la socialisation 
professionnelle des nouveaux actifs, comme support d'identité, ainsi que comme espace social d'innovation (Lucas, 2005 ; Mundler et Rémy, 2012).

L'effacement de la famille comme composante sociale centrale de la production agricole peut-elle être un des facteurs explicatifs de l'actuelle réorganisation sociale et professionnelle de l'agriculture ? Cette hypothèse laisse entrevoir l'action collective de proximité comme l'un des nouveaux lieux de socialisation, d'innovation et de projet en agriculture, dont les activités se distribuent à des degrés divers entre l'exploitation et différentes formes d'organisation collective. Dans ce cadre, l'activité agricole devient un objet de moins en moins saisissable par les outils actuels des politiques publiques fondées sur le chef d'exploitation et l'unicité de l'exploitation.

\section{Comment évaluer la dimension coopérative ?}

La coopération agricole de production repose sur des modalités d'organisation informelles et formelles, et parmi ces dernières, toutes ne se caractérisent pas par un statut juridique d'ordre associatif ou coopératif. Face à la complexité et l'hétérogénéité des formes possibles de coopération agricole de production, il apparaît difficile d'avancer une définition normative, caractérisant une fois pour toute sa dimension coopérative. L'enjeu de cette caractérisation étant avant tout institutionnelle, notamment au regard de l'action publique, il apparaît plus judicieux d'avancer vers un faisceau de critères, à l'exemple d'autres expériences tentées soit en agriculture (Gasselin, 2011) ou dans l'économie sociale (Draperi, 2011). La finalité est ainsi plus de produire une grille d'analyse pour interroger chaque forme sociale et ainsi l'évaluer, sur sa dimension coopérative et ses plus-values vis-à-vis de la collectivité. Ceci permettrait alors à terme de justifier de soutiens publics spécifiques (fiscalité avantageuse, aides financières, accès aux marchés publics, etc.).

\section{Repenser l'accompagnement}

L'hétérogénéité des formes sociales et techniques d'agriculture et de leurs logiques complique les processus de coordination entre les producteurs, qui peuvent nécessiter de nombreuses concertations pour mettre les besoins et projets en adéquation avec les objectifs de l'action commune. Or, les études démographiques montrent que le renouvellement des actifs agricoles sera désormais davantage le fait de jeunes aux profils diversifiés (niveaux supérieurs d'étude, avec plus d'expériences hors agriculture et plus d'installations hors cadre familial, etc.), phénomène susceptible de renforcer l'hétérogénéité des agriculteurs, de leurs profils et de leurs logiques (Lefebvre, 2009 ; Wepierre et al., 2012). Par ailleurs, les capacités d'accompagnement pour faciliter et rendre plus efficace l'action collective des agriculteurs sont aujourd'hui fragilisées : d'une part, par la diminution des financements publics aux organismes d'appui aux groupes, et d'autre part, par manque de compétences adéquates pour rendre possible l'action commune entre la diversité des agriculteurs (Ruault et Lémery, 2009 ; Compagnone et al., 2013). 
Au regard de ces constats, l'investissement de l'action publique, à travers la recherche et le développement agricole, reste plus que jamais nécessaire afin de contribuer à renforcer les compétences d'accompagnement des processus de coordination entre agriculteurs.

\section{Conclusion}

Le paysage de l'action collective de proximité se recompose sous l'effet des nouveaux défis économiques et agro-écologiques rencontrés par les agriculteurs, et des mutations sociales et territoriales qui affectent le secteur. Au contraire des périodes précédentes, les nouvelles formes de coopération entre agriculteurs sont plus autonomes et endogènes et peu dictées par les opérateurs de l'encadrement agricole, les dispositifs de l'action publique ou les mots d'ordre syndicaux. La coopération agricole de production analysée dans cet article atteste d'un élargissement du champ de la mutualisation des activités et des investissements agricoles et transforme les frontières et le fonctionnement de l'exploitation, qu'il s'agisse de l'organisation du travail, des formes juridiques, du projet patrimonial ou encore de la prise de décision. Ces mutations remettent en cause les outils de l'action publique et des organisations agricoles articulés autour de l'objet « exploitation agricole ». Elles appellent entre autres, à renouveler les problématiques de recherche scientifique : où sont les frontières de ces nouvelles formes de coopération ? Quelles sont les modalités et les compétences d'accompagnement nécessaire à leur consolidation ? S'agit-il seulement de nouvelles formes d'organisation de la production ou plus radicalement de nouveaux modèles de production ? Sont-elles de nouvelles expressions de la coexistence et de la confrontation des modèles agricoles à l'échelle territoriale ?

C'est à une partie de ces questions qu'un nouveau projet de recherche-action impulsé par la FN Cuma et l'Inra, en partenariat avec d'autres acteurs ${ }^{8}$, va chercher à répondre dans les années à venir pour aller plus loin dans la compréhension approfondie de ce phénomène, et mettre à l'épreuve les hypothèses explicatives émises dans les pages précédentes.

\section{Bibliographie}

ACI, 1996. Déclaration sur l'identité coopérative internationale, Congrès de Manchester 1995, Alliance coopérative internationale, Genève.

Allaire, G., Boyer, R., 1995. La grande transformation de l'agriculture : Lectures conventionnalistes et régulationnistes, Paris, INRA/Economica.

8. Coop de France, FNAB, FN Civam, Gaecs et Sociétés, Trame, Supagro Florac, École supérieure d'agriculture d'Angers. 
Allaire, G., Fuzeau, V., Therond, O., 2013. La diversité des cultures et l'écologisation de la Politique agricole commune. Recherches en économie et sociologie rurale, INRA Sciences sociales, $\mathrm{n}^{\circ}$ 1-2.

Barraud-Didier, V., Henninger, M.-C., Anzalone, G., 2012. La distanciation de la relation adhérent-coopérative en France, Études rurales, 190, 119-130.

Barry, C., 2012. Commercialisation des produits agricoles : Un producteur sur cinq vend en circuit court, Agreste Primeur, 275.

Barthez, A., 2000. Le droit comme expression culturelle. Processus de légalisation du travail familial en agriculture : le cas du GAEC, Revue de droit rural, 621-632.

Bonnieux, F., 2009. Bilan critique de la politique agri-environnementale et perspectives d'évolution, in Aubert, F., Piveteau, V., Schmitt, B. (Eds), Politiques agricoles et territoires, Versailles, éditions Quae, 141-164.

Bouba-Olga, O., Grossetti, M., 2008. Socio-économie de proximité, Revue d'Économie Régionale \& Urbaine, 3, 311-328.

Charroin, T., Veysset, P., Fromont, J.-L., Devienne, S., Palazon, R., Ferrand, M., 2012. Productivité du travail et économie en élevages d'herbivores : définition des concepts, analyse et enjeux, Productions Animales, 25, 193-210.

Cochet, H., 2008. Vers une nouvelle relation entre la terre, le capital et le travail, Études foncières, 134, 24-29.

Compagnone, C., Lémery, B., Petit, S., Kockmann, F., Moretty, P., 2013. Forme et réforme organisationnelles des Chambres d'agriculture. Une lecture à partir des régimes d'action des conseillers, Économie Rurale, 337, 41-58.

Cordellier, S., 2014. Une histoire de la coopération agricole de production en France, RECMA, 331.

Darré, J.-P., 1996. L'invention des pratiques dans l'agriculture : vulgarisation et production locale de connaissance, Paris, Karthala.

De Torcy, B., Pommereul, P., 2012. Groupes de développement agricole : intérêts et limites des formes actuelles, Journées d'étude sur la coopération agricole de production, FN Cuma, Paris, 26 avril.

Demeulenaere, É., Goulet, F., 2012. Du singulier au collectif, Terrains \& travaux, 1, 20, 121-138.

Draperi, J.-F., 2011. L'économie sociale et solidaire, une réponse à la crise ? Capitalisme, territoires et démocratie, Paris, Dunod.

Fiamor, A.-E., 2011. Valorisation alimentaire et développement local. Analyse à partir d'un exemple drômois, Anthropology of food, 8. [online] URL : http:// aof.revues.org/6797\#quotation

Figureau, E., 2013. Évaluation et conception de céréales à paille à usage fourrager, Mémoire de fin d'études d'ingénieur, ENSAT, Toulouse.

Filippi, M., Frey, O., Mauget, R., 2009. Les coopératives agricoles face à l'internationalisation et la mondialisation des marchés, RECMA, 310, 31-51.

Fleury, P., Chazoule, C., Peigné, J., 2011. Agriculture biologique et agriculture de conservation : ruptures et transversalités entre deux communautés de pratiques, Colloque Les transversalités de l'agriculture biologique, SFER, Strasbourg, 23-24 juin. 
FN Cuma, 2012a. Coopération agricole de production, FN Cuma, Paris.

FN Cuma, 2012b. Projet politique 2012, FN Cuma, Paris.

FN Cuma, 2013. CAP Vert-Développer les pratiques agroécologiques grâce à de nouvelles coopérations à la production en agriculture. CASDAR - Appel à projets d'innovation et de partenariat 2013, ministère de l'Agriculture.

Garambois, N., Devienne, S., 2012. Les systèmes herbagers économes. Une alternative de développement agricole pour l'élevage bovin laitier dans le Bocage vendéen ? Économie rurale, 330-331, 56-72.

Gasselin, P., 2011. Co-conception d'une politique publique pour une région arrière du développement : le cas de la pluriactivité en Languedoc-Roussillon, POUR, 212, 155-163.

Gasselin, P., Barbier, J.-M., Touzard, J.-M., 2012a. La « plus-value » du groupe : regards croisés, Journées d'étude sur la coopération agricole de production, FN Cuma, Paris, 12 Juin.

Gasselin, P., Vaillant, M., Bathfield, B., 2012b. The activity system. A position paper, $10^{\text {th }}$ European IFSA Symposium, Aarhus, 1-4 July.

Gasselin, P., Bathfield, B., 2013. La flexibilidad de los sistemas de actividad : un marco de análisis de las propiedades y de los procesos adaptativos de las actividades de los agricultores, in Gasselin, P., Cloquell, S., Mosciaro, M. (Eds.), Adaptación y Transformaciones de las Agriculturas Pampeanas al Inicio del Siglo XXI, Buenos Aires, Ciccus, 281-314.

Giddens, A., 1987. La constitution de la société. Éléments de la théorie de la structuration, Paris, PUF.

Hervieu, B., 2002. La multifonctionnalité de l'agriculture : genèse et fondements d'une nouvelle approche conceptuelle de l'activité agricole, Cahiers Agricultures, 11, 6, 415-419.

Hirczak, M., 2007. La co-construction de la qualité agroalimentaire et environnementale dans les stratégies de développement territorial. Une analyse à partir des produits de la région Rhône-Alpes, Thèse en géographie, Université Joseph Fourier, Grenoble.

Hostiou, N., Fagon, J., 2012. Simplification des conduites d'élevage : analyse transversale des pratiques mises en œuvre dans les filières herbivores et granivores, Productions animales, 25, 127-140.

Lacam, M., Thomas, F., Valorge, F., Vaquié, P.-F., 2012. Coopération agricole de production : de nouvelles marges de manœuvre pour les agriculteurs, Colloque Diversité et durabilité des modèles agricoles coopératifs dans un contexte de crises de la mondialisation, SFER, Paris, 6-7 novembre.

Lamine, C., Bellon, S., 2009. Transitions vers l'agriculture biologique : pratiques et accompagnements pour des systèmes innovants, Versailles, Quae.

Le Guen, R., 2011. Pour une sociologie compréhensive de la profession agricole, Mémoire de HDR, Université de Nantes.

Le Guen, R., de Casinière, H., 2010. Approche socio-documentaire de la méthanisation dans le Grand-Ouest de la France, Rapport d'étude BIODEOCL2 PSDR-GO, 185 p. 
Lefebvre, F., 2009. Démographie agricole : la France doit-elle craindre l'avenir ? Prévisions à l'horizon 2020, Déméter, 223-248.

Lefevre, D., 1996. À l'ombre des machines: Les Cuma, 50 ans de solidarités locales, Paris, Éditions Entraid.

Léger, F., 2013. Collective action on agro-ecological practices and public goods management in France : from theory to reality. European Conference Let's work together : territorial cooperation for the provision of public goods in the context of the reformed CAP, Groupe de Bruges, Bergerie de Villarceaux, 20-21 décembre.

Lémery, B., 2003. Les agriculteurs dans la fabrique d'une nouvelle agriculture, Sociologie du Travail, 45, 1, 9-25.

Lémery, B., 2011. Les agriculteurs : une profession en travail, in Béguin, P., Dedieu, B., Sabourin, E. (Eds), Le travail en agriculture : son organisation et ses valeurs face à l'innovation, Paris, L'Harmattan, 243-254.

Le Velly, R., Bréchet, J.-P., 2011. Le marché comme rencontre d'activités de régulation : initiatives et innovations dans l'approvisionnement bio et local de la restauration collective, Sociologie du Travail, 53, 4, 478-492.

Lucas, V., 2005. Coopératives agricoles et développement durable : le défi du renouvellement de l'engagement coopératif, Mémoire du Master Professionnel DEES, IEP-UPMF, Grenoble.

Lucas, V., 2012. Des groupes d'échange pour accompagner la transition en agriculture de conservation. Rapport final - Tâche 8 du Projet PEPITES, UMR Innovation, Montpellier.

Lucas, V., 2013. Coopération agricole de production et Agroécologie, Rapport final Mission d'étude, FN Cuma, Paris.

Lurois, E., 2012. Une coopérative d'activités agricoles au service de l'emploi et des territoires, Journées d'étude sur la coopération agricole de production, FN Cuma, Paris, 4 septembre.

Mahé, T., Lerbourg, J., 2012. Des agriculteurs bio diplômés, jeunes et tournés vers les circuits courts, Agreste Primeur, 284.

Mazoyer, M., Roudart, L., 2002. Histoire des agricultures du monde : du néolithique à la crise contemporaine. Paris, Seuil.

Meynard, J.-M., Messéan, A., Charlier, A., Charrier, M., Farès, M., Le Bail, M., Magrini, M.-B., Savini, I., 2013. Freins et leviers à la diversification des cultures - Etude au niveau des exploitations agricoles et des filières, Synthèse du rapport d'étude DGPAAT n 10-18, INRA, Paris.

Mondy, B., Terrieux, A., Thomas, F., (à paraître). Des premières Cuma de diversification aux ATC, vers une évolution des formes coopératives de production ?, in Mundler, P., Valorge, F., Mondy, B., Couzy, C., Ateliers de transformation collectifs. Transformer collectivement ses produits agricoles dans les territoires.

Moraël, P., 2012. Coopératives agricoles, installation et territoire, Journées d'étude sur la coopération agricole de production, FN Cuma, Paris, 4 septembre.

Mundler, P., Rémy, J., 2012. L'exploitation familiale à la française : une institution dépassée ? L'Homme et la société, 183-184, 161-179. 
Mundler, P., Valorge, F., Mondy, B., Couzy, C., (à paraître). Ateliers de transformation collectifs. Transformer collectivement ses produits agricoles dans les territoires.

Nicolas, P., 1988. Émergence, développement et rôle des coopératives agricoles en France. Aperçus sur une histoire séculaire, Économie rurale, 184, 116-122.

Nil, A., 2012. Étude de faisabilité d'un projet d'organisation interprofessionnelle pour la filière luzerne, Rapport CGAAER n 11173, Conseil général de l'alimentation, de l'agriculture et des espaces ruraux, Paris.

Ostrom, E., 1990. Governing the Commons : The Evolution of Institutions for Collective Action, Cambridge, Cambridge University Press.

Pierre, G., 2013. Produire pour son territoire. De l'autonomie agricole au projet collectif agro-énergétique. Illustrations dans l'Ouest français, Mémoire de HDR, Université de Caen-Basse-Normandie.

Ploeg, J.-D. van der, 2014. Les paysans du $21^{e}$ siècle. Mouvements de repaysannisation dans l'Europe d'aujourd'hui, Paris, ECLM.

Ploeg, J.-D. van der, Laurent, C., Blondeau, F., Bonnafous, P., 2009. Farm diversity, classification schemes and multifunctionality, Journal of Environnemental Management, 90 (2), 124-131.

Quentin, N., 2012. Résultats de l'observatoire technico-économique du RAD. Synthèse 2012 - Exercice comptable 2011, RAD, Cesson-Sévigné.

Ricci, P., Bui, S., Lamine, C., 2011. Repenser la protection des cultures : innovations et transitions, Dijon, Educagri.

Rip, A., Kemp, R., 1998. Technological Change, in Rayner, S., Malone, E. (Eds), Human Choice and Climate Change, Colombus, Battelle press, 327-399.

Ruault, C., Lémery, B., 2009. Le conseil de groupe dans le développement agricole et local : pour quoi faire et comment faire ? in Compagnone, C., Auricoste, C., Lémery, B. (Eds), Conseil et Développement En Agriculture : Quelles Nouvelles Pratiques? Quae/Educagri, 71-96.

Sabourin, É., 2007. L'entraide rurale, entre échange et réciprocité, Revue du MAUSS, $30,198-217$.

Sabourin, É., 2012. Organisations et sociétés paysannes : une lecture par la réciprocité, Versailles, Quae.

Séronie, J.-M., Boullet, P., 2007. L'exploitation agricole flexible, Les cahiers CER France, CER France.

Touzard, J.-M., Draperi, J.-F., 2003. Les coopératives entre territoires et mondialisation, Paris, L'Harmattan.

Tritz, Y., 2012. Le Système énergétique agri-territorial : les bioénergies comme outil de développement local, Géographie, économie, société, 14, n $131-52$.

Vanloqueren, G., Baret, P.-V., 2009. How agricultural research systems shape a technological regime that develops genetic engineering but locks out agroecological innovations? Research Policy, 38, n 6, 971-983.

Wepierre, A.-S., Lerbourg, J., Courleux, F., 2012. Un agriculteur sur cinq a moins de quarante ans, Agreste Primeur, 293. 